\title{
The Effect of Luminance Condition on Form, Form-from-Motion and Motion Perception
}

\author{
Giovagnoli Sara ${ }^{1,}$, , Pansell Tony ${ }^{2}$, Bolzani Roberto ${ }^{1}$, Hellgren Kerstin ${ }^{2}$, Benassi Mariagrazia ${ }^{1}$ \\ ${ }^{1}$ Department of Psychology, University of Bologna, Bologna, Italy \\ ${ }^{2}$ Department of Clinical Neuroscience, Karolinska Institutet, Bernadottelaboaratoriet, S: T Eriks Ögonsjukhus, Stockholm, Sweden
}

\section{Email address:}

sara.giovagnoli@unibo.it (G. Sara)

${ }^{*}$ Corresponding author

\section{To cite this article:}

Giovagnoli Sara, Pansell Tony, Bolzani Roberto, Hellgren Kerstin, Benassi Mariagrazia. The Effect of Luminance Condition on Form, Formfrom-Motion and Motion Perception. American Journal of Applied Psychology. Vol. 6, No. 6, 2017, pp. 158-165.

doi: 10.11648/j.ajap.20170606.14

Received: March 25, 2017; Accepted: April 8, 2017; Published: November 8, 2017

\begin{abstract}
This study investigated to what extent rod-dominated vision affects motion and form perception accuracy. Twenty-nine healthy subjects took part in the experiment. Form coherence (FC), form-from-motion (FFM) and motion coherence (MC) tests were assessed in low-light (rod-dominated vision) and high-light (cone-dominated vision) conditions. For each test we determined the accuracy by evaluating the correct detection obtained in five levels of coherence (corresponding to different signal-to-noise ratio). The results evidenced that motion, form and form-from-motion accuracy decreased in low-light condition. Furthermore, light condition effect was differently mediated by noise according to the type of task. The motion perception is affected only at high noise levels, while form discrimination was globally affected at all the levels, also in absence of noise, both for static (FC) and dynamic stimuli (FFM). We conclude that in rod-dominated vision form-from-motion perception is more defected than form and motion perception. We hypothesized that our results are due to the integration between $\mathrm{M}$ and $\mathrm{P}$ cells in FFM test increases the form perception accuracy in high-light condition but this advantage is completely lost during low-light condition, when the rods need to integrate information both from $\mathrm{M}$ and P cells.
\end{abstract}

Keywords: Low-Light Level, Form Perception, Form-from-Motion Perception, Motion Perception, Coherence

\section{Introduction}

Visual function in humans is strongly reduced at low luminance levels, such as at night. Several studies demonstrated that when the luminance level is very low, under scotopic visual condition, visual acuity (spatial resolution) becomes worse, color discrimination decreases radically, contrast sensitivity is reduced and temporal resolution is compromised. However, the mechanisms underlying how low-light condition affects vision system functionality have still to be clarified. The vast majority of the studies proposed models of visual system based on findings obtained from high luminance level experiments $[1$, $2,3,4,5,6]$. Only in the last decades the researchers' interest has been focalized on vision at very low luminance condition and this could allow to enrich the knowledge on the vision and redefine the current visual system models.
The human visual system is primarily organized as to be most effective in diurnal, photopic luminance condition. As referring to the widely acknowledged visual system model, the external world perception, the recognition of objects, their colors and their movement depends on two anatomically and functionally separated visual pathways that work in parallel and elaborate the visual information from the retina to the brain $[1,2,3,4,5,6]$. The Parvocellular pathway (Ppathway) is sensitive to high spatial and low temporal frequencies stimuli and it projects mainly into the temporal lobes via the ventral stream. The Magnocellular pathway (Mpathway) is sensitive to low spatial and high temporal frequencies and it is primarily connected to the parietal lobes via the dorsal stream [7]. This stream is highly sensitive to motion and low luminance signal.

Despite the evidences confirming their segregation, recent findings $[8,9,10]$ attest the relevance of the interaction between the dorsal and the ventral stream using stimuli 
which enhance both M- and P-pathways, such as form-frommotion and biological motion stimuli. These stimuli require the integration of the motion perception to detect the form of an object. Several neuropsychological studies have shown that the perception of form created from moving stimuli (form-from-motion) is mediated by specific neural pathways $[8,9,10]$, involving the connections between the M- and Psystems. It is indeed likely that form-from-motion perception can persist despite impaired perception of static form or motion [for a review see ref. 11]. Deficit in form-frommotion perception has been demonstrated in subjects having form and motion perception preserved [8, 9, 10]. Other studies have described subjects having deficits in motion perception but with preserved form and form-from-motion perception [12, 13, 14] as well as have been documented cases where a deficit in static form perception saw unaltered first order motion and form-from-motion perception [14]. This results underline that the ability to extract form-frommotion is not derived from simple summation of the information from form and motion but depends on the functioning of larger neural networks. Some authors hypothesized that this complex neural network depends on a connection, described as a 'crosstalk', between the two visual pathways $[8,11]$.

Physiological evidences indicated that under scotopic luminance levels the activation of $\mathrm{P}$ cells is drastically reduced and the rods' input to $\mathrm{P}$ cells is very weak, in contrast $\mathrm{M}$ cells are able to maintain high levels of activation $[15,16,17]$. Therefore, it is plausible to argue for the central role of $\mathrm{M}$ pathway in scotopic luminance condition, not excluding the role, albeit weak, of the $\mathrm{P}$ pathway and the possible inter-connection of the two visual pathways.

Form perception has rarely been studied in condition of low-light level, such as in low mesopic or scotopic conditions $[18,19]$. The biological motion perception was studied by different scientific authors $[18,19,20]$. All the cited studies show that both form and biological motion perception are highly defected in scotopic conditions. The form-frommotion perception in low-light condition was tested by Takeuchi, Yokosawa and De Valois [21] and by Grossman and Blake [19]. In Grossman and Blake study [19] the subjects were asked to discriminate the orientation, vertical or horizontal, of a rectangle defined by moving dots against a differently moving background. The authors speculated that the worse performance in form-from-motion perception in scotopic condition is due to rods' poor spatial pooling. The excessive proximity and the inadequate dimensions of the target-stimuli could make the distinction between the signals difficult, losing the configurable structure of the images created by adjacent stimuli.

On the other hand, several studies have investigated motion perception in very low-luminance levels (i.e. in roddominated vision) and have demonstrated that motion perception is affected in scotopic light level [18, 19, 20, 22, $23,24,25,26,27]$. However, the rod disadvantage in motion perception seemed to rely on the difficulty in extracting temporal changes. This disadvantage was primarily confirmed by speed discrimination deficits emerged clearly when the velocity of the moving stimuli was faster than $4 \%$ sec. [26, 28]. Billino, Bremmer, and Gegenfurtner [20] speculated that the higher threshold for motion and radial flow perception in scotopic vision was mainly due to rods' weak temporal pooling, decreasing the ability of motion detection at high velocities and complex velocity discrimination (i.e. temporal summation). This issue has also been confirmed by recent findings [18] measuring steady state visual evoked potentials. However, when considering global motion detection, involving high order motion processing (MT area), at a relatively low velocity (from $3.2 \% \mathrm{sec}$ to $8.0^{\circ} / \mathrm{sec}$ ), failed to confirm a clear motion deficit under scotopic condition [19]. In a forerunner study Orban, de Wolf and Maes [29] have demonstrated a highest impairment at very low temporal frequencies.

These findings, albeit controversial [20], left open a major question: "Which type of motion or form stimuli could preserve perception at low-light level?" To our best knowledge these doubts are still not resolved. Little is known concerning if the spatial pooling related to global motion perception could interfere or aid motion perception, probably also because all the studies investigating motion perception under scotopic vision calculated the psychophysical threshold to compare low-light to high-light motion detection performance, while no one evaluated how the noise could affect the motion direction discrimination.

In the present study, we aim to analyze and compare the score profiles and thresholds to investigate how the luminance condition can affect the noise effect on form, form-from-motion recognition and motion direction discrimination at low-light level (presumably as scotopic condition, or rod-dominated vision) as compared to highlight level (photopic condition, considered as conedominated vision).

\section{Methods}

\subsection{Participants}

Twenty-nine healthy subjects participated in the experiment, 25 females (mean age $=25.5 ; \mathrm{SD}=3.7$ ) and 4 males (mean age=23.4; $\mathrm{SD}=4.1$ ). The regional ethics committee approved the research protocol and all subjects gave their informed consent. The research has been carried out in accordance with The Code of Ethics of the World Medical Association (Declaration of Helsinki). The privacy rights of subjects have been observed. All subjects had normal or corrected-to-normal visual acuity ( $\log M A R \leq 0.0$; ETDRS Sloan Letter Chart, Precision Vision).

\subsection{Stimuli}

Three different perceptual tests were used: the Motion Coherence (MC) test in order to evaluate the motion discrimination ability, the Form Coherence (FC) test to measure the form discrimination ability and the Form-frommotion (FFM) test to evaluate the ability in recognizing a 
form created by moving dots. All three stimuli have a common appearance: all of them are displayed on a Sony CPD-300SFT computer monitor (20" CRT monitor; vertical refresh rate $150 \mathrm{~Hz}$ ) at an eye to screen distance of 60 centimeters. They are composed of white dots displayed on a dark background; each stimulus is created within a circular frame subtending $9.4^{\circ}$ visual angle, presented at the center of the computer screen. The luminance of the high-light stimulus was measured on a large $\operatorname{dot}\left(3^{\circ}\right)$ displayed on the screen using a Hanger Universal Photometer S4. For the low light stimulus, the luminance has been measured in the same way by means of a linear photodiode. In the two light conditions, the luminance of the target stimuli has been modified without changing the other stimuli's characteristics. In this paper, we refer to two light levels as 'high' and 'low' because we didn't use artificial pupils and we didn't measure the pupil's diameter, therefore, it wasn't possible specify the exact retinal illumination associated with these luminance conditions. However, based on our results and on the luminance of the stimuli it is reasonable to consider that our 'high-light' level is a photopic visual condition and the 'lowlight' level is a scotopic visual condition. In the high-light condition the dot luminance was $42 \mathrm{~cd} / \mathrm{m}^{2}$, while in low-light condition the dot luminance was equal to $0.00961 \mathrm{~cd} / \mathrm{m}^{2}$. The luminance contrast between background and stimuli was kept constant at $93 \%$ in both conditions. In addition, each test had its specific characteristics described in the following paragraphs (see paragraphs Form Coherence Test, FormFrom-Motion Coherence Test and Motion Coherence Test).

\subsubsection{Form Coherence Test}

The Form Coherence (FC) test [30] is composed by 1050 static dots (3 pixels diameter) presented in the center of the screen within a circular frame. The dots' position within the frame determines a spatial pattern (i.e. each dot has the equal horizontal and vertical distance from the others) that configures a recognizable form (see Figure 1). Eight different figures are used as target-forms and they are chosen because they are simple enough to be recognizable without too many details. The eight figures are balanced between four geometric/abstract shapes (circle, square, triangle and star) and four concreate/easily recognizable figures (house, bear, doll and cup). The test is composed of five coherent levels. The coherence is obtained by the number of aligned dots (i.e. each dot has the same distance from the others), whereas the noise is given by the randomly positioned dots. In the first level (0-level) all the dots constituting the form are coherently aligned and only the dots outside the form are randomly positioned ( $100 \%$ coherence, $0 \%$ of noise). In each subsequent level the coherence within the form decreases by two decibels (36.9\%) making form recognition more difficult. The signal-to-noise ratio is inversely related to the level of difficulty of the task (0-level: $100 \%$ of coherence; level 1: $63.1 \%$; level 2: $39.8 \%$; level 3: $25.1 \%$; level 4: $15.8 \%$ ). In each level eight trials are presented and the eight forms selected are randomly chosen. The stimulus is displayed for $3000 \mathrm{msec}$ before it disappears. The subject's task was to identify the form and verbally indicate the perceived shape. To dampen the undesirable effect of reduced motivation and attention when increasing the test difficulty, a 0 -level stimulus was added in each noise level (level 1-4). The total number of repetitions for the FC-test was 44 (12 at 0 -level and 8 in each subsequent level). The correct responses in each difficulty level have been used as a measure of form discrimination ability.

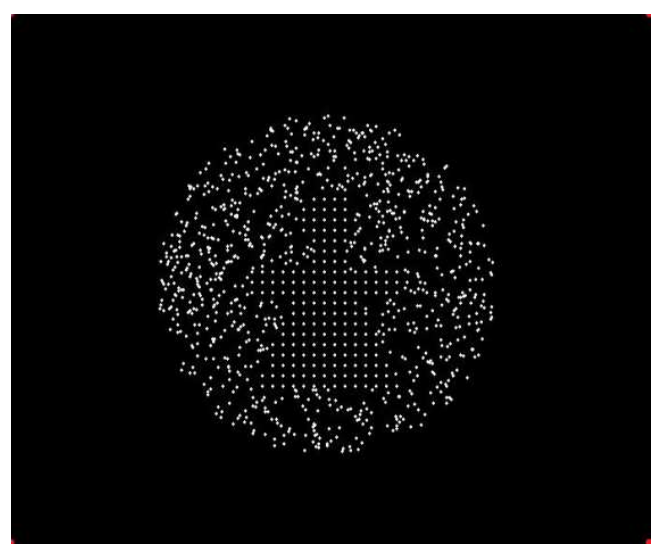

Figure 1. Form Coherence stimulus. The aligned dots define the form to be recognized.

\subsubsection{Form-from-Motion Coherence Test}

The test has the same characteristics as the FC-test for luminance, number of dots, stimuli dimensions, forms and difficulty levels but in the FFM-test the forms are created by coherently moving dots instead of coherently aligned static dots. The dots outlining the form (including those in the shape) move coherently to the right, while all the other dots outside are static (see Figure 2). Five coherence levels are presented. In each subsequent level the signal-to-noise ratio is reduced by two decibels (i.e. the number of coherent moving dots outlining the form is reduced, and the noise increases making the task more difficult). At the 0-level, $100 \%$ of the dots outlining the form move coherently to the right ( $100 \%$ coherence, $0 \%$ noise). At level $1,63.1 \%$ of the dots continue to moving toward the right, while $36.9 \%$ of the dots become static. For each subsequent levels of difficulty (level 2, 3 and 4) $36.9 \%$ of the moving dots become static (the coherence levels are the same as the FC test; see paragraph Form Coherence Test).

The dots are moving at constant velocity of $8 \% \mathrm{sec}$ (11 pixels per frame). The subjects had to recognize and verbally report the perceived form, choosing between eight possible shapes (i.e. circle, square, triangle, star, house, bear, doll or cup). To dampen the undesirable effect of reduced motivation and attention when increasing the test difficulty, a 0-level stimulus was added in each noise level (level 1-4). Each subject performed 44 trials, eight repetitions on five difficulty levels plus four extra 0-level stimuli for enhanced motivation. The correct responses in each difficulty level have been used as a measure of FFM discrimination ability.

Usually form-from-motion task is made up of dots moving in a specific direction or having a specific speed inside the 
shape while dots outside the form are moving in the opposite direction or having a different speed. Therefore, to perceive the form by motion, the subject has to extract information about direction or speed of motion.

In our task, it is only required to perceive the presence of motion to detect the form and this might contribute to make the FFM task easier.

We decided to keep the dots outside the form steady to make the difference between the FC and the FFM test only on how the form is defined: by the spatially coherent dots in FC and by the moving dots in FFM test. In the MC test, we did not use a steady background because the contrast is not between moving and not moving dots but between coherently and non-coherently moving dots (see paragraph Motion Coherence Test).

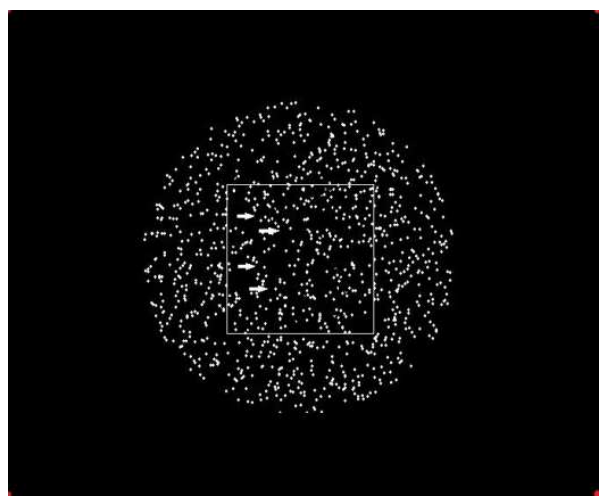

Figure 2. Form-from-Motion stimulus. The dots inside the form (here a square drawn for clarity but not shown during the test) move coherently while the dots outside are steady.

\subsubsection{Motion Coherence Test}

The MC test is a modified version of Motion Coherence Test used in previous studies [30, 31]. The MC test consists of 120 dots ( 5 pixels diameter; $0.12^{\circ}$ visual angle at $60 \mathrm{~cm}$; see Figure 3) moving coherently in one of eight directions: four cardinal (up, right, down and left) and four oblique (upright, down-right, down-left and up-left). The dots move at a constant velocity of $4.4^{\circ} / \mathrm{sec}$ ( 6 pixel per frame) coherently in one of the eight direction of the space or in a Brownian manner. Hammett and colleagues [28] have described distorted speed perception at speeds above $4 \%$, whereas speed perception at lower speeds has been found unaltered. Each dot has a limited lifetime of $200 \mathrm{msec}$ (equal to 6 animation frames) to avoid visual tracking. The stimulus lasted for $2000 \mathrm{msec}$. The MC test consists of five coherence levels where the coherence was given by the percent of coherent moving dots. In each coherence level the subject performs 8 trials, one for each coherent direction randomly selected. The test starts with $100 \%$ of coherence (0-level) and the signal-to-noise ratio decreases exponentially by two decibels for each level (36.9\%). The coherence levels are the same as the FC and FFM tests (0-level: 100\% of coherence; level 1: 63.1\%; level 2: 39.8\%; level 3: $25.1 \%$; level 4: $15.8 \%)$. To dampen the undesirable effect of reduced motivation and attention when increasing the test difficulty, a 0 -level stimulus is added on each noise level (level 1-4).
Each subject performed 44 trials, eight repetitions on five difficulty levels plus four extra 0-level stimuli for enhanced motivation. The subject was asked to verbally indicate the direction of coherently moving dots to indicate the perceived stimulus movement direction. The number of correct detection in each level was used as a measure of motion perception accuracy.

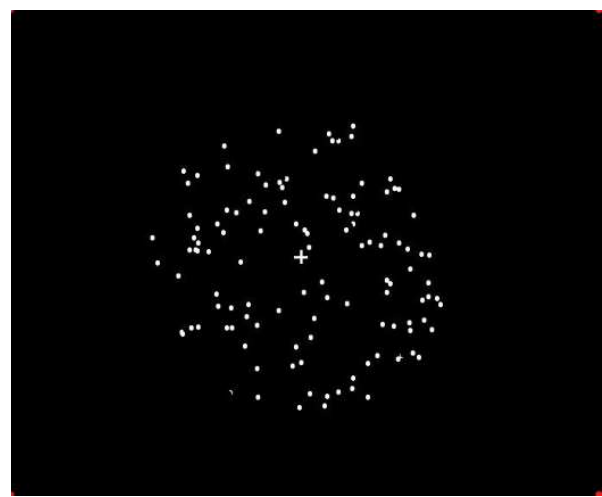

Figure 3. Motion Coherence stimulus. The bright dots are coherently moving in a circle area on a dark background. A central cross was used for visual fixation.

\subsubsection{Low Contrast Visual Acuity Testing.}

To ensure that no subject had relevant problems or deficit in contrast sensitivity a low-contrast $(1.25 \%)$ visual acuity test was performed using the Sloan Letter Chart (ETDRS) in the standardized luminance cabinet (Precision Vision) on $2 \mathrm{~m}$ eye-chart distance.

\subsection{Procedure}

According to the experimental protocol, all the subjects started with a demonstration-test to familiarize with the task. The demonstration consisted of 16 trials at different coherence levels. The subjects were then directed to protocol 1 or 2 to balance for light condition (high/low). Protocol 1 started with the low-light condition tests while protocol 2 started with the high-light condition tests.

Before performing the tests in the low-light condition, the subject had to stay in a completely darkened room for 20 minutes to permit visual dark adaptation. After 20 minutes the test started according to the test protocol. To allow complete darkness except for the stimuli on the screen, a CRT monitor was used, connected across the wall of the room with an external computer, managed by the researcher. The subject was sitting in front of the screen approximately at $60 \mathrm{~cm}$ eye-screen distance.

The researcher checked the stimuli on the CRT monitor and the subjects gave responses via a microphone. The responses from each test were immediately recorded in an Excel file. After the low-light condition, the low-contrast sensitivity was evaluated and, after 20 minutes, the subjects repeated the three tests in the high-light condition. All the tests were administered in the same room with the same test equipment. In the high-light condition the room was uniformly illuminated. 
In order to have the same timing between the tasks also in the second protocol the subjects had the same time intervals between a task and the other. The protocol 2 began with the low-contrast sensitivity test, then high-light condition, and finally the low-light condition.

The perceptual tests were administered with two different sequences to alternate the tests order among the subjects. The first sequence began with FC-test, then the FFM-test and last the MC-test. The second sequence began with MC-test, then the FFM-test and last the FC-test. During all experiments, viewing was tested with unaffected pupils.

\subsection{Data Analysis}

In the data analysis, each test is treated separately to compare the subjects' performances in the two visual conditions (Condition: high vs low). A Repeated Measures Analysis of Variance was applied to compare the percentage of correct responses considering the high and low-light conditions and the five coherence levels (Level: 100\%, 63,1\%, 39,8\%, $25,1 \%$ and $15,8 \%$ ) as within-subject factors separately for each test (Test: MC-test, FC-test and FFM-Test).

To analyze the change between the high-light and the lowlight condition, we estimated the accuracy in low-light condition at the same coherence level producing an accuracy equal to $70 \%$ in high-light condition. The difference between the accuracy obtained in low-light condition and the $70 \%$ reference value was used as measure of the change between the two visual conditions. To estimate the accuracy, the psychophysical curve was evaluated by fitting the data with the error function. A Repeated Measures Analysis of Variance was applied to compare the score change calculated for each test as within-subject factors (Test: MC-test, FC-test and FFM-Test).

Finally, to understand how the performance obtained in FFM-test is linked or depends on the motion perception or the form perception abilities, a Pearson correlation analysis has been done to analyze the relation between the total scores obtained in three tests (Test: MC-test, FC-test and FFM-Test) in high-light condition.

\section{Results}

\subsection{Form Coherence Test}

The form recognition was affected by luminance level (High-/low-light condition effect: $\mathrm{F}_{1,28}=39.587 ; \mathrm{p}<.001$; Partial Eta Squared=.586): the global accuracy obtained in high-light condition (mean $=.662, \mathrm{sd}=.022$ ) was better than in low-light condition (mean=.469, sd=.024). The accuracy in form perception was also related to the presence of noise (Coherence level effect: $\mathrm{F}_{4,25}=102.627$; $<$ <.001; Partial Eta Squared $=.943$ ), showing that the accuracy decreased with noise increment (see Figure 4). Moreover, the accuracy was relatively more affected by noise in the high-light than in the low-light condition (High-/low-light condition by Coherence level effect: $F_{4,25}=5.604 ; p=.002$; Partial Eta Squared=.473). According to the noise increment, the form recognition accuracy decreased steeper in high-light than in low-light condition.

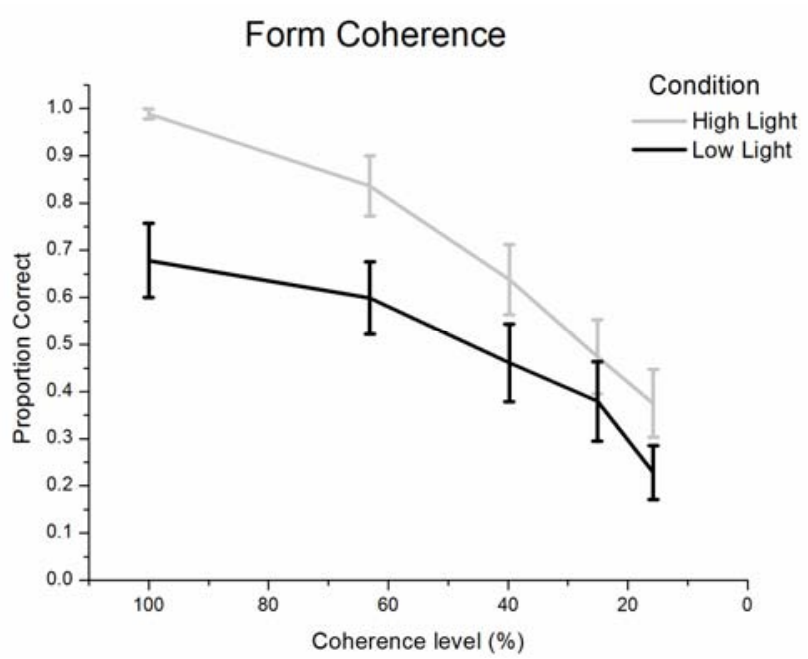

Figure 4. Form Coherence score: mean scores obtained in each coherent level in high light condition (grey lines) and in low light condition (black lines). The error bars represent the $95 \%$ confidence intervals.

\subsection{Form-from-Motion Coherence Test}

The results showed that luminance affected form perception obtained in FFM task (High-/low-light condition effect: $\mathrm{F}_{1,28}=424.823 ; \mathrm{p}<.001 ;$ Partial Eta Squared=.938); the accuracy score obtained in high-light condition (mean=.954; $\mathrm{sd}=.013$ ) was significantly better than in low-light condition (mean=.373; sd=.025). The accuracy decreased with noise increment (Coherent level effect: $F_{4,25}=60.459 ; p<.001$; Partial Eta Squared=.906). Moreover, accuracy was more affected by noise in rod dominated vision as compared to cones' (High/low-light condition by Coherence level effect: $F_{4,25}=36.572$; $\mathrm{p}<.001$; Partial Eta Squared=.854). As could be seen in Figure 5 the reduced accuracy by increasing noise level, differed strongly between the two light conditions. While in the highlight condition the noise influence was visible only in the two last levels (level 3 and 4), in the low-light condition the accuracy worsened starting from the 0 -level.

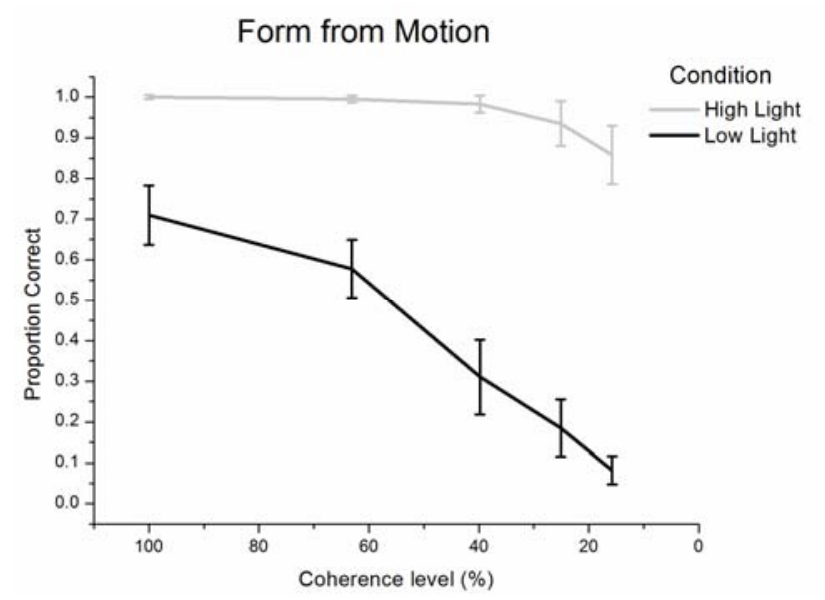

Figure 5. Form-from-Motion score: mean scores obtained in each coherent level in high light condition (grey lines) and in low light condition (black lines). The error bars represent the $95 \%$ confidence intervals. 


\subsection{Motion Coherence Test}

The results showed no differences between the motion perception accuracy obtained in high and low luminance level (High-/low-light condition effect: $\mathrm{F}_{1,28}=3.436$; $\mathrm{p}=.074$; Partial Eta Squared=.109); the accuracy score obtained in high-light condition (mean=.771; sd=.032) was almost the same as the accuracy obtained in the low-light condition $($ mean=.725; $\mathrm{sd}=.032)$. The accuracy decreased with noise increment (Coherence level effect: $\mathrm{F}_{4,25}=58.882 ; \mathrm{p}<.001$; Partial Eta Squared=.904). In the high-light condition the accuracy was more affected by noise than in the low-light condition (Luminance condition by Coherence level effect: $\mathrm{F}_{4,25}=4.455 ; \mathrm{p}=.007$; Partial Eta Squared=.416). However, observing the accuracy means calculated for each noise level in the two visual conditions (Figure 6), the obtained performance was almost the same in the first three noise levels (0-level, level 1 and level 2), and only in level 3 and 4 the accuracy decreased in low-light condition.

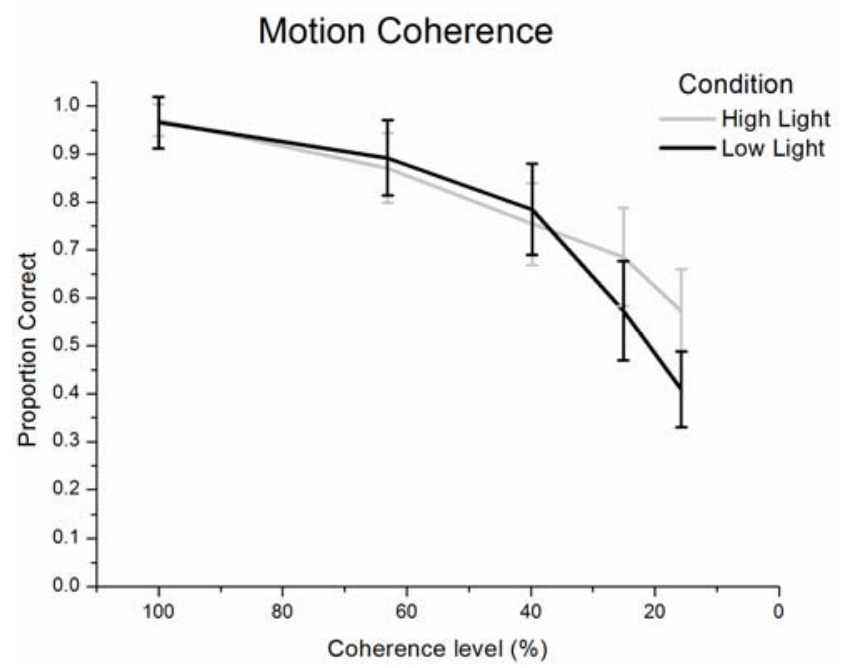

Figure 6. Motion Coherence score: mean scores obtained in each coherent level in high light condition (grey lines) and in low light condition (black lines). The error bars represent the $95 \%$ confidence intervals.

\subsection{The Score Changes}

The three stimuli changes between the accuracy obtained in low- and high-light condition have been compared by an ANOVA for repeated measures. More negative are the scores and higher is the difference detected. The overall difference between the stimuli score changes is significant $\left(\mathrm{F}_{2,27}=72.138, \mathrm{p}<.001\right.$; Partial Eta Squared=.842). The multiple contrasts analysis showed that the form change is significantly higher than the motion change $\left(\mathrm{F}_{1,28}=4.209\right.$, $\mathrm{p}=.049$; Partial Eta Squared=.131) and the FFM change is significantly higher than both the motion and the form changes $\left(\mathrm{F}_{1,28}=128.422, \mathrm{p}<.001\right.$; Partial Eta Squared=.821). However, the dispersion of the values around the median in FFM score change (visible observing the FFM box in Figure 7) is strongly lower respect to the dispersion visible in $\mathrm{MC}$ and in FC box (see Figure 7).

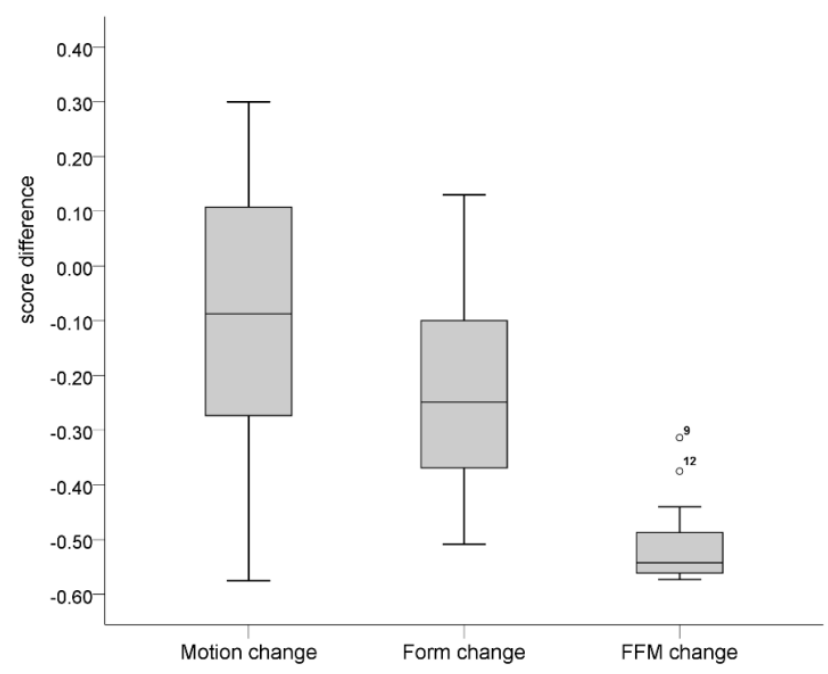

Figure 7. Score changes between high-light and low-light condition evaluated at the noise reference value corresponding to the $70 \%$ of the correct answers in high-light condition. The box represents the interquartile range (from 25 th to 75 th percentile), the line inside shows the median.

\subsection{Pearson Correlation Analysis}

The Pearson correlation analysis has been done using the total scores obtained in each test in high-light condition. The results showed no significant correlation between MC-test score and FFM-test score $(r=.079 ; \mathrm{p}=.682)$. A significant correlation has been found between the score obtained in FFM-test and that obtained in FC-test $(r=.403 ; \mathrm{p}<.001)$. No significant correlation has been found between $\mathrm{MC}$-test and FC-test $(\mathrm{r}=.217 ; \mathrm{p}=.258)$.

\section{Discussion}

The general goal of the present study was to evaluate form recognition and motion perception under high- and low-light condition by evaluating the effect of noise in a form discrimination task (which stimulates predominantly the Ppathway), in a form-from-motion task (that depends on the interaction of $\mathrm{P}$ - and $\mathrm{M}$-pathways) and in motion perception task (which is supposed to stimulate predominantly the Mpathway).

The results not only confirmed worse performances in low-light conditions in form and form-from-motion perception $[19,21]$ but also showed a worse performance even in absence of noise, demonstrating a very poor connection between rods and P- cells in low-light condition.

Furthermore, the obtained results showed that in the highlight condition the form perception seems to take advantage by moving stimuli as compared to static ones. In FFM test the highest performance was maintained with the coherence at $100 \%$ (0-level), 63.1\% (level 1) and at 39.8\% (level 2), whereas both the $\mathrm{MC}$ and $\mathrm{FC}$ performances decreased already on first level (63.1\%). This FFM advantage in highlight condition may be a result of the high efficiency of cones in the integration of M- and P-pathways information due to the cones' good connections with $\mathrm{M}$ - and P-cells [32]. The photopic condition advantage was completely lost in low- 
light condition where the FFM performance was strongly reduced.

In the FFM test, the moving stimuli increase the form boundaries recognition, enhanced by temporal pooling which allows the integration of dots in a coherent contour. In the low-light condition, the loose of cones activity [17], determines disrupted rods' input to FFM's neural circuit [8] that interferes with form recognition. The discrimination of a form created by motion implies two different tasks. First, to perceive the motion of the stimuli, and secondly to determine the contour of the area delimiting the moving stimuli. In other words, FFM perception requires the recognition of the shape of the motion zone. If the cones are not activated or are weakly activated the form recognition is deficient because of the poor connections of the rods with the P-system. It could be hypothesized that the signal is disrupted both at low (within the connection between rods, $\mathrm{M}$ and $\mathrm{P}$ cells) and at high level of visual processing (cross-talk within extrastriate cortex involving both ventral and dorsal areas). The drastic reduction of the performance, in a task that requires information on stimuli motion to recognize a form, could be the result of an impaired activation (or a non-activation) of the complex neural networks allowing the integration between $\mathrm{M}$ and $\mathrm{P}$ pathway.

The difficulty in form perception under rod dominated vision is confirmed also for static stimuli. The form recognition created by coherently aligned static dots is simply obtained by spatial pooling and segregation between coherent and noise dots. In the low-light condition, the form perception based on static stimuli was significantly worse as compared to the high-light condition, confirming the results of Purpura, Kaplan and Shapley [17].

Differently from the FC and FFM, the MC accuracy measured as direction discrimination is not significantly different between the high and low-light levels. This result is in accordance with Grossmann and Blake results [19] and with the hypothesis that the M-pathway could dominate visual perception in scotopic luminance levels [15, 17, 33]. It is possible that motion perception performance would decrease differently using a higher stimuli velocity, as other studies have previously shown [20,28].

It is worth to note that the MC stimuli velocity was above $4 \% \mathrm{sec}$ allowing to eventually find differences in motion perception between rod- and cone-dominated vision [20, 26]. The accuracy was almost the same in the two light conditions when considering the coherence levels from 100\% till $39.8 \%$, but when the noise further increase the performance dropped faster in the low- than in high-light condition. Thus, our results showed that low-light condition affect motion perception only at low levels of coherence. This result has never been discussed before because other findings did not compare the effect of low luminance on different noise levels.

Finally, the performance worsening observed in low-light level, compared to that obtained in high-light level, appears more relevant in FC and FFM test than in MC test. This finding is confirmed by the score change analysis. The score changes are significantly different in the three tests. In particular, the change detected for FFM-test is significantly higher than those evaluated for FC and for MC-test as well as the $\mathrm{FC}$ score change is significantly higher than the $\mathrm{MC}$ score change. Furthermore, the dispersion of the values around the median in FFM score change is strongly lower than the dispersion found in MC and in FC (see Figure 7).

Analyzing the relation between the scores obtained in the three different tasks, it is possible to see that the performance obtained in the FFM-test is correlated with that obtained in FC-test but not with that obtained in MC-test. In other words, it seems that the relations between the three performances concern the type of task (identification of a form in FC and FFM tests), more than the type of stimuli (moving dots in FFM and MC tests).

It's important to mention that the two visual conditions used in the experiment were called 'high-light' and 'lowlight' condition because the authors were unable to specify the exact retinal illumination associated with these light conditions. However, based on our results and on the luminance of the stimuli, it is reasonable to consider that our 'high-light' level is a photopic condition (cone-dominated vision) and the 'low-light' level is a scotopic condition (roddominated vision).

\section{Conclusion}

Our results confirmed general worse performances in lowlight conditions in FC, MC and FFM. However, the formfrom-motion perception in rod-dominated vision is more defected than form and motion perception. The integrated work of $\mathrm{M}$ and $\mathrm{P}$ cells, required to perceive a form created by moving stimuli, produces an advantage in high-light condition but this advantage is completely lost in low-light condition. We hypothesize that the drastic reduction of the performance observed in rod-dominated vision could be the result of an impaired activation of the complex neural networks allowing the integration between $\mathrm{M}$ and $\mathrm{P}$ pathway due to the weakly activation of the cones in low-light condition and the poor connections of the rods with the Psystem.

\section{References}

[1] Nassi, J. J., \& Callaway, E. M. (2009). Parallel processing strategies of the primate visual system. Nature Reviews. Neuroscience, 10(5), 360-372.

[2] Milner, A. D., \& Goodale, M. A. (2008). Two visual systems re-viewed. Neuropsychologia, 46, 774-785.

[3] Orban, G. A. (2008). Higher order visual processing in macaque extrastriate cortex. Physiological Reviews, 88(1), 5989.

[4] Goodale, M. A., \& Milner, A. D. (1992). Separate visual pathways for perception and action. Trends in Neurosciences, 15(1), 20-25. 
[5] Livingstone, M. \& Hubel, D. (1988). Segregation of form, color, movement, and depth: anatomy, physiology, and perception. Science, 240(4853), 740-749.

[6] DeYoe, E. A., Van Essen, D. C. (1988). Concurrent processing streams in monkey visual cortex. Trends in Neurosciences, 11(5), 219-226.

[7] Maunsell, J. H. R., Nealey, T. A., \& DePriest, D. D. (1990). Magnocellular and parvocellular contributions to responses in the middle temporal visual area (MT) of the macaque monkey. Journal of Neuroscience, 10, 3323-3334.

[8] Blanke, O., Brooks, A., Mercier, M., Spinelli, L., Adriani, M., Lavanchy, L., et al. (2007). Distinct mechanisms of formfrom-motion perception in human extrastriate cortex. Neuropsychologia, 45, 644-653.

[9] Cowey, A., \& Vaina, L. M. (2000). Blindness to form from motion despite intact static form perception and motion detection. Neuropsychologia, 38, 566-578.

[10] Regan, D., Giaschi, D., Sharpe, J. A., \& Hong, X. H. (1992). Visual processing of motion-defined form: Selective failure in patients with parietotemporal lesions. The Journal of Neuroscience, 12(6), 2198-2210.

[11] Matheson, H. E., \& McMullen, P. A., (2010). Neuropsychological dissociations between motion and form perception suggest functional organization in extrastriate cortical regions in the human brain. Brain and Cognition 74, $160-168$.

[12] Vaina, L. M., Cowey, A., LeMay, M., Bienfang, D. C., \& Kikinis, R. (2002). Visual deficits in a patient with 'kaleidoscopic disintegration of the visual world'. European Journal of Neurology, 9(5), 463-477.

[13] McLeod, P., Dittrich, W., Driver, J., Perrett, D., \& Zihl, J. (1996). Preserved and impaired detection of structure from motion by a 'motion-blind' patient. Visual Cognition, 3(4), 363-391.

[14] Vaina, L. M. (1994). Functional segregation of color and motion processing in the human visual cortex: Clinical evidence. Cerebral Cortex, 4(5), 555-572.

[15] Lee, B. B., Smith, V. C., Pokorny, J., \& Kremers, J. (1997). Rod Inputs to Macaque Ganglion Cells. Vision Research, 37(20), 2813-2828.

[16] Lennie, P., \& Fairchild, M. D. (1994). Ganglion cell pathways for rod vision. Vision Research, 34, 477-482.

[17] Purpura, K., Kaplan, E., \& Shapley, R. M. (1988). Background light and the contrast gain of primate $\mathrm{P}$ and $\mathrm{M}$ retinal ganglion cells. Proceedings of the National Academy of Sciences U.S.A., 85, 4534-4537.

[18] Burton, E., Wattam-Bell, J., Rubin, G. S., Atkinson, J., Braddick, O., Nardini, M. (2016). Cortical processing of global form, motion and biological motion under low light levels. Vision Research, 121, 39-49.

[19] Grossman, E. D., \& Blake, R. (1999). Perception of coherent motion, biological motion and form-from-motion under dimlight conditions. Vision Research, 39(22), 3721-3727.

[20] Billino, J., Bremmer, F., \& Gegenfurtner, K. R. (2008). Motion processing at low light levels: Differential effects on the perception of specific motion types. Journal of Vision, $8(3): 14,1-10$.

[21] Takeuchi, T., Yokosawa, K., \& De Valois, K. K. (2004). Texture segregation by motion under low luminance levels. Vision Research, 44(2), 157-166.

[22] Duffy, K. R. \& Hubel, D. H. (2007). Receptive field properties of neurons in the primary visual cortex under photopic and scotopic lighting conditions. Vision Research, 47, 2569-2574.

[23] Takeuchi, T., De Valois, K. K., \& Motoyoshi, I. (2001). Light adaptation in motion direction judgments. J. Opt. Soc. Am. A, 18(4), 755-764

[24] Gegenfurtner, K. R., Mayser, H., \& Sharpe, L. T. (2000). Motion perception at scotopic light levels. Journal of the Optical Society of America A, Optics, Image Science, and Vision, 17, 1505-1515.

[25] Takeuchi, T. \& De Valois, K. K. (1997). Motion-reversal Reveals Two Motion Mechanisms Functioning in Scotopic Vision. Vision Research, 37(6), 745-755.

[26] Takeuchi, T. \& De Valois, K. K. (2000). Velocity discrimination in scotopic vision. Vision Research, 40(15), 2011-2024.

[27] Takeuchi, T. \& De Valois, K. K. (2009). Visual motion mechanisms under low retinal illuminance revealed by motion reversal. Vision Research, 49(8), 801-809.

[28] Hammett, S. T., Champion, R. A., Thompson, P. G., \& Morland, A. B. (2007). Perceptual distortions of speed at low luminance: Evidence inconsistent with a Bayesian account of speed encoding. Vision Research, 47, 564-568.

[29] Orban, G. A., de Wolf, J., \& Maes, H. (1984). Factors influencing velocity coding in the human visual system. Vision Research, 24(1), 33-39. http://dx.doi.org/10.1016/00426989(84).

[30] Giovagnoli, S., Benassi, M., Hellgren, K., Forsman, L. \& Bolzani, R. (2014). The relation between acuity of the Approximate Number System and dorsal and ventral stream functions. Journal of Vision, 14, 804.

[31] Menghini, D., Finzi, A., Benassi, M., Bolzani, R., Facoetti, A., Giovagnoli, S., Ruffino, M., \& Vicari, S. (2010). Different underlying neurocognitive defects in developmental dyslexia: a comparative study. Neuropsychologia, 48, 863-872.

[32] Schwartz, S. H. (2009). Visual Perception: A Clinical Orientation, Fourth Edition: A Clinical Orientation, Fourth Edition. New York: McGraw Hill Professional.

[33] Lee, B. B., Pokorny, J., Smith, V. C., Martin, P. R., \& Valberg, A. (1990). Luminance and chromatic modulation sensitivity of macaque ganglion cells and human observers. Journal of the Optical Society of America A, 7(12), 2223-2236. 